\title{
Structure and Response in the World Trade Network
}

\author{
Jiankui He and Michael W. Deem \\ Departments of Bioengineering and Physics \& Astronomy, Rice University, Houston, Texas 77005, USA
} (Received 18 June 2010; revised manuscript received 13 August 2010; published 5 November 2010)

\begin{abstract}
We examine how the structure of the world trade network has been shaped by globalization and recessions over the last 40 years. We show that by treating the world trade network as an evolving system, theory predicts the trade network is more sensitive to recessionary shocks and recovers more slowly from them now than it did 40 years ago, due to structural changes in the world trade network induced by globalization. We also show that recession-induced change to the world trade network leads to an increased hierarchical structure of the global trade network for a few years after the recession.
\end{abstract}

DOI: 10.1103/PhysRevLett.105.198701

PACS numbers: 89.65.Gh, 89.75.Hc

The physical theory of evolution predicts that under certain conditions, a changing environment leads to the development of modular structure [1-3]. The prediction depends only on (i) the dynamics of the response to change being "slow" due to a glassy landscape, (ii) the presence of change, and (iii) the exchange of information between evolving agents. Since the trade network is an evolving system, this physics of evolution may be applied to the world trade system, previously studied by network analysis [4-6]. We assume that condition 1 is satisfied for the world trade network due to the complexities of intercountry relationships. Condition 2 is satisfied by viewing recessions as causing a change of the environment for the dynamics of the world trade system. Condition 3 is satisfied because information flow naturally results from transfer of business practices or material between countries. Thus, the theory of $[1,2]$ allows us to make three predictions: decreased modular structure in the world trade network increases the sensitivity to recessionary shocks, decreased modular structure decreases the rate of recovery, and recessions themselves spontaneously increase the modular structure of the world trade network. All three predictions will be borne out by data. These results are general predictions about how the detailed structural parameters of the evolving economic system will organize. Our theory shows that the modular and hierarchical structure formed in response to environmental fluctuation increases the resistance to and rate of recovery from perturbations. The theory predicts that globalization, which reduces hierarchical structure, should lead to increasingly large recessions and a decreased rate of recovery, in contrast to standard economic understanding [7].

To apply the physical theory of evolution that describes the spontaneous emergence of modularity in fluctuating environments [1,2] to world trade, we seek a mathematical representation of hierarchy in the world trade network. Identification of network motifs or modules is an active research field in the physics of networks [1,8,9], with the study of structure at multiple scales, i.e., hierarchy, somewhat more recent $[10,11]$. In this Letter, we treat the world trade data as defining a geometry in trade space. We project the trade topology onto the best treelike topology representing the data. The success of this projection in representing the original geometry is used to define the hierarchy of the original data.

We apply hierarchical clustering to construct the best treelike representation of the world trade network. Correlation between the distances implied by the tree construction and the distances defined by the original trade data is calculated. This quantity is termed the cophenetic correlation coefficient (CCC) [12]. We will display the general trend of the CCC since 1969, noting especially the increase of the CCC after each recession. The magnitude of the CCC will be shown to correlate with the ability of total world GDP to resist a recessionary shock. Theory shows that this result is, in fact, causal, not simply a correlation, which is a major result here.

We focus on how global recessions, such as the 20082009 recession, have affected the structure of the world trade network. Modular structures arise in the trade network, for example, because countries in a trade group trade among themselves to a greater extent than with others. These trade groups may interact with each other to form higher level groupings. The detailed reasons for an increase of hierarchy in the world trade network are many: perhaps protectionism for the domestic economy [13], or because long-distance trade seems costly during a recession. Standard arguments in economic theory suggest a decreased rate of recovery from recession for trade networks with more modular structure [7]. We will see, however, that our theory predicts that greater trade network structure increases both the resistance to recessionary shocks and the rate of recovery from recessions.

A hierarchical trade network occurs when countries with strong trade connections group into trade modules or regional trade clusters. A flat or nonhierarchical structure occurs when countries trade evenly with all other countries, and there are no regional trade modules in the trade network. We use the historical trade data from the United Nations database (Comtrade) from 1962 to 2007. We build the world trade network with nodes representing countries 
and links representing the trade value. We do not scale the trade volume by the GDP, because small economic units should not have the same weight as large economic units. First, a distance matrix is calculated from the trade network matrix by $d_{i j}=M^{*}-M_{i j}$, where $M^{*}=\max \left(M_{i j}\right)$. Here, $M_{i j}$ is the trade value between two countries. The average linkage hierarchical clustering algorithm is applied to the distance matrix to produce the treelike dendrogram [12]; see Fig. 1. We define the treelike structure to have the most hierarchy. Therefore, the amount of hierarchy can be measured by the likeness between the original data and the best tree that is produced from the original data by hierarchical clustering. The CCC quantifies this likeness. The cophenetic matrix is generated from the dendrogram. Its elements are the branch distance where two objects become members of the same cluster in the dendrogram: for two nodes, $i j$, we find the nearest common bifurcation point, and the branch length for this point is the cophenetic element of these two nodes, $c_{i j}$; see Fig. 1 for an example. The CCC is defined as CCC $=\left[\sum_{i<j}\left(d_{i j}-\bar{d}\right) \times\right.$ $\left.\left(c_{i j}-\bar{c}\right)\right] / \sqrt{\left[\sum_{i<j}\left(d_{i j}-\bar{d}\right)^{2}\right]\left[\sum_{i<j}\left(c_{i j}-\bar{c}\right)^{2}\right]}$, where $d_{i j}$ and $\bar{d}$ are the element and average of elements of the distance matrix, and $c_{i j}$ and $\bar{c}$ are the elements and average of elements of cophenetic matrix, respectively. Hierarchical data sets have a high CCC value, and nonhierarchical data sets have a low CCC value [14].

A major factor affecting the world trade network over the last 40 years has been the process of globalization. Qualitatively, this globalization has been expressed as a "flattening" of the world [15]. Here, we use the CCC to measure how the hierarchical structure of the world trade network has changed over time. Large CCC values indicate
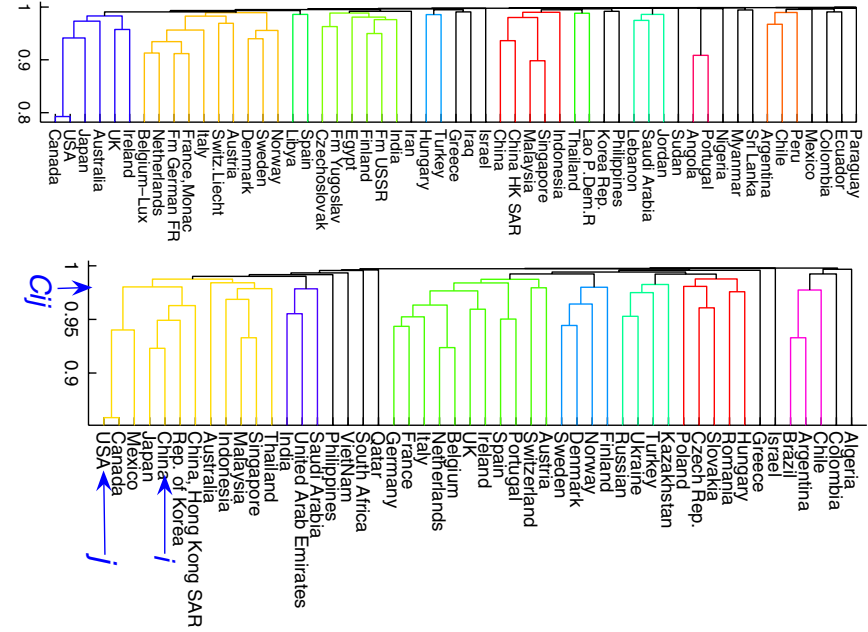

FIG. 1 (color online). Dendrogram representation of trade networks for selected countries at 1969 (top figure) and 2007 (bottom figure). Trade modules are marked by different colors. The structure of trade networks has changed greatly from 1969 to 2007. We plot only selected countries, because the figure becomes crowded if all countries are plotted. higher hierarchy. The major trend of CCC with time in Fig. 2 is a reduction of hierarchy as the flattening has taken place [15]. We notice, however, that the CCC does not always decrease year by year. We notice that during and after each recession, marked on the figure, the CCC value increases. The CCC values at the year after recession are larger than that at the year before the recession ( $p$ value $=0.003$ of the Kolmogorov-Smirnov test for null hypothesis that they are from the same distribution with the same mean, and $p$ value $=0.0006$ for null hypothesis that $\mathrm{CCC}$ value before recession is larger than that after recession). This trend is true both for the past 3 major recessions and for the past 4 minor recessions. The scale of increase of the hierarchical structure depends on the severity of recession. One possible reason for this CCC trend during recessions is the increase of trade protectionism during recessions. Also, regional integrations are greatly enhanced during recessions, leading to increased regional imports [16], which strengthens trade modules. Free trade promotes globalization and decreases the hierarchy of the trade networks. But trade protectionism and regional integration, which is common during recessions to protect domestic or regional economies by restraining trade between countries, tends to reduce trade between countries in different trade modules. Thus, recessions may promote the regionalization that enhances the modularity of the trade network. One example is the Asian currency crisis of 1997, which lead to the development of independent Asian monetary systems.

The CCC is a characterization of the world trade network that is independent from the total amount of world trade. In the process of globalization, a country tends not only to increase its total trade value, but also to trade with more

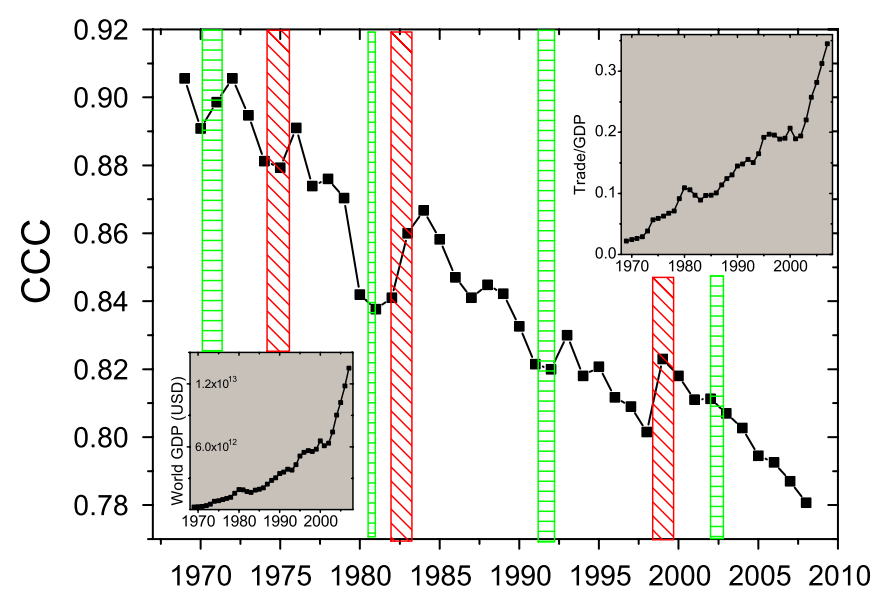

FIG. 2 (color online). The CCC from 1969 to 2007. Data from 2008 are not yet available. Shaded rectangles marked the seven recessions. Left and right borders are positioned at the start and end of a recession, respectively, according to U.S. National Bureau of Economic Research. During sharp recessions, the CCC increases significantly, and during mild recessions, the CCC usually increases mildly. The upper right inset is the ratio of total world trade to world GDP. The lower left inset is the total world trade in units of U.S. dollar. 


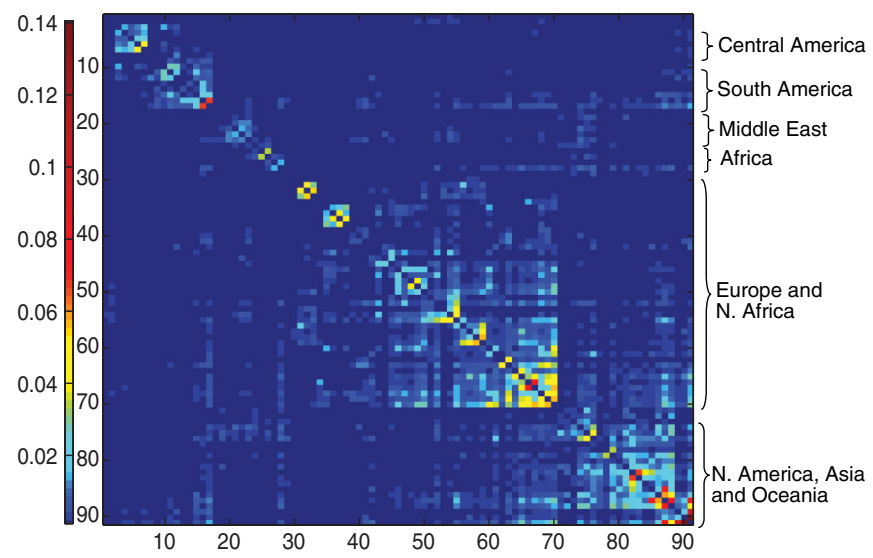

FIG. 3 (color online). The trade share matrix $S_{i j}=$ $M_{i j} /\left(\sum_{m=1}^{N} M_{i m}+\sum_{n=1}^{N} M_{j n}\right)$ after hierarchical clustering between countries in 2007. We can see clearly several modules: North American + Asian + Oceanic countries, European + North African countries, Middle and South Africa, Middle East, South America, and Central America.

partners. The upper right inset of Fig. 2 shows the typically increasing ratio of world trade to GDP. Only the recessions of 1981, 1991, 1997, and 2001 lead to a decrease in the trade to GDP ratio, whereas the CCC increased in all seven recessions. The increased hierarchical structure appearing after all seven recessions in Fig. 2, is therefore, a sensitive correlate of recessions, and independent of the trade to GDP ratio shown in the upper right inset of Fig. 2 and total trade volume shown in the lower left inset of Fig. 2. Measurement of globalization by both hierarchical structure (CCC) and total trade provides complementary information.
The CCC quantifies the development of hierarchical structure in the trade network at multiple scales in an integrated way. The clustering of the world trade network shows the modularity of global trade; see Fig. 3. The development of regional trading partners occurs simultaneously with globalization. By comparing the structure of trade network in 1969 and in 2007, we found that the increased trade among Canada, the United States, and Mexico as a result of NAFTA is one example of a regional trading group. Regional trade pacts among the Middle East countries are other examples of regionalization. In general, free trade markets will develop modular structure at multiple geographical scales.

The ability of the trade system to respond to recessionary perturbations is proportional to the hierarchical structure present, i.e., it increases with the CCC value, according to the evolutionary theory of modular structure development $[1,2]$. That is, the modular structure that exists at multiple scales affects how recessions propagate in the trade network, just as modular structure of person-to-person contacts affects how diseases spread in a population. We examine how the network structure affects the propagation of a recession throughout the world. For example, if there is a $1 \%$ decrease of the GDP of the USA, by how much does the total GDP of the world, excluding the USA, decrease due to the spread of recession from the USA? We investigate the five most recent global recessions including the 2007-2009 crisis. We calculate the ratio of GDP change (percentage) of the world excluding the USA to the GDP change (percentage) of the USA in each recession as a function of the CCC value in each recession; see Fig. 4(a). We observe that in more recent
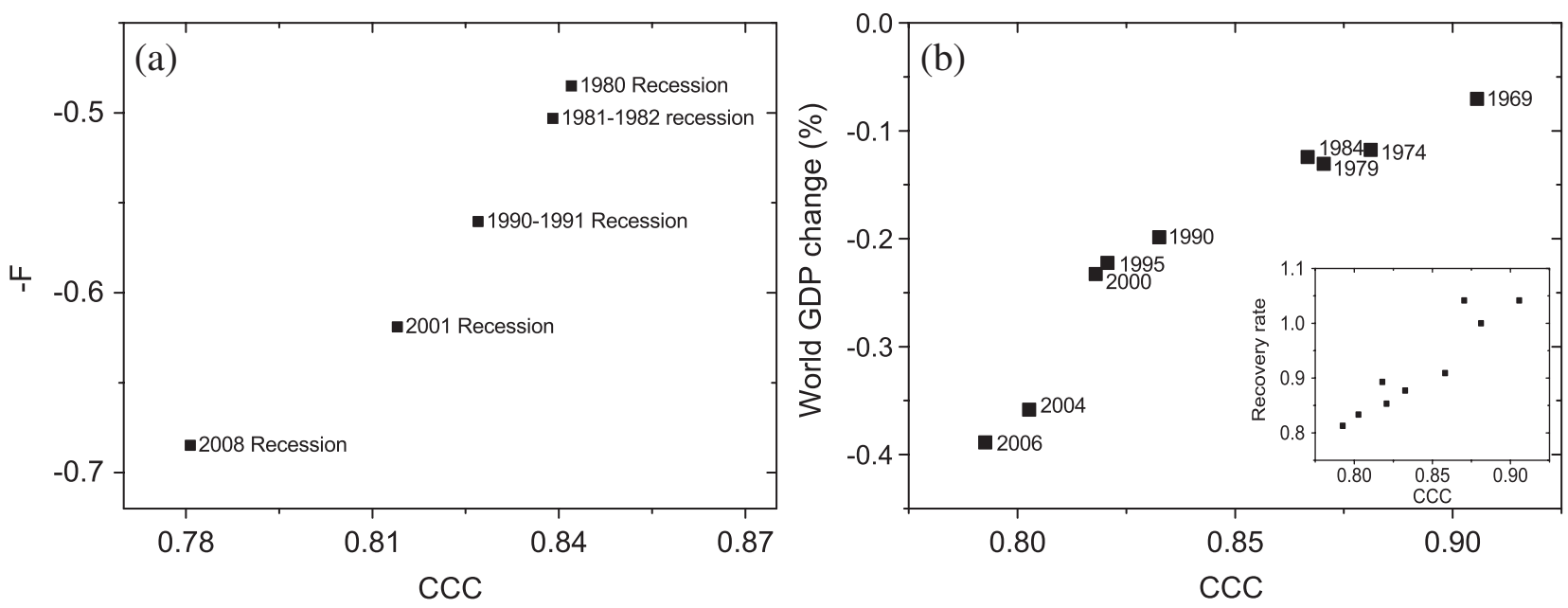

FIG. 4. (a) The ratio of the total world excluding the USA GDP change (percentage) to the change of the USA GDP (percentage) in 5 recessions, $F$ in the $y$ axis. The GDP change in one recession is defined as the GDP decline from peak to trough. Quarterly GDP data are used to find the peak of recession. The quarterly GDP data for 2001 and 2008 recessions are from [21]. The quarterly GDP data for earlier recessions are estimated from annual GDP data. (b) Impulse response analysis of spread of recession. In each year, initial values are set to historical trade and GDP data. A recession is assumed to begin in the USA and spread to the rest of the world. The world GDP change is plotted as a function of the CCC. The reduction in the world GDP is greater when the CCC value is low. Inset figure: The GDP recovery from recession can be well fit by the relation $Y(t) \sim Y(\infty)-a \exp (-\lambda t)$. Yearly recovery rates, $\lambda$, are shown versus the CCC. In accord with theory, the recovery rate is positively correlated with the CCC. 
recessions with less hierarchical structure of the trade network, a recession in the USA has a stronger impact on the rest of the world. This result indicates a strong positive correlation between lack of hierarchical structure and severity of recession impact.

We also perform an impulse response analysis of the vector autoregression (VAR) model to analyze the time evolution of recession [17-19]. We explore the possible underlining causal links between lack of hierarchical structure and severity of recession. A recession is assumed to start in the USA. The U.S. GDP is initially reduced by the maximal GDP decline during the recession, e.g., the maximal quarterly U.S. GDP decline was $5.4 \% \mathrm{~S}$ in the 2008 2009 recession [20]. The export from country $i$ to country $j, X_{i j}$, is updated by a factor of ratio of GDP of country $j$ at time $t, Y_{j}(t)$, and $\left.t-1, Y_{j}(t-1)\right)$ [19]. Thus, $X_{i j}(t)=$ $X_{i j}(t-1) Y_{j}(t) / Y_{j}(t-1)$. Then the GDP of country $i$ is updated by $Y_{i}(t+1)=Y_{i}(t)+P_{i}\left(X_{i}(t) / X_{i}(t-1)-1\right)$. Where $P_{i}=X_{i} / Y_{i}$ is the ratio of export to GDP for country $i$. The GDP of each country decreases until steady state is reached, at which point the simulation is terminated. We calculate the world GDP change as $\left(\sum_{i} Y_{i}^{\text {steady }}-\right.$ $\left.\sum_{i} Y_{i}^{\text {initial }}\right) / \sum_{i} Y_{i}^{\text {initial }}$. We observe how the crisis spreads globally and is measured the GDP loss during crisis.

The impulse response analysis results support that the severity of the 2008-2009 recession may be due to loss of hierarchical structure in the global trade network. Lack of hierarchical structure makes the world trade network less resistant to recession, as observed from Fig. 4(b). We believe this increased sensitivity is due to a loss of modular or hierarchical structure in the world trade network; see Fig. 2. As an example, the impact of a recession on the GDP is more severe in 2006 than in 1968, by a factor of 5.7 . Interestingly, after this calculation was carried out, an estimate of the ratio of the reduction of GDP in 2009 to the average reduction over past recessions equaling 6 was reported [20].

Evolutionary theory has shown that systems under environmental perturbation not only increase their modularity, but also increase their response function to perturbations $[1,2]$. In the present context, this would imply that as trade has been globalized, and the CCC reduced, the rate of recovery from recession should decrease. We consider this phenomenon in the world trade network, using the VAR model. After the system reaches steady state following the reduction to the USA GDP, we impose a positive impulse to restore the USA GDP to its initial value. The world GDP recovers, at a rate that depends on the hierarchical structure of the trade network. We observe that when the trade network has greater hierarchical structure, indicated by a larger CCC value, the trade network recovers more quickly from recession, as shown in the inset figure of Fig. 4(b).

We have used the concept of viewing the world trade network as defining a geometry in trade space and the idea of projecting this geometry to the best treelike topology to define the hierarchy in the world trade network. With that necessary mathematical prolegomena, we introduced the world trade network as an evolving system. Physics of evolution in changing environments was then used to predict that the world trade network is more sensitive and recovers more slowly from evolutionary shocks now than it did 40 years ago, because globalization has reduced hierarchical structure in the world trade network. We also predict that recession-induced change to the world trade network should lead to a temporarily increased hierarchical structure of the global trade network. These predictions, contrary to standard economic thinking, were borne out by our study of the world trade data since 1969 .

[1] J. Sun and M.W. Deem, Phys. Rev. Lett. 99, 228107 (2007).

[2] J. He, J. Sun, and M. W. Deem, Phys. Rev. E 79, 031907 (2009).

[3] N. Kashtan and U. Alon, Proc. Natl. Acad. Sci. U.S.A. 102, 13773 (2005).

[4] C. Hidalgo, B. Klinger, A. Barabasi, and R. Hausmann, Science 317, 482 (2007).

[5] M. Barigozzi, G. Fagiolo, and D. Garlaschelli, Phys. Rev. E 81, 046104 (2010).

[6] P. B. Slater and W. Schwartz, IEEE Trans. Syst. Man Cybern. SMC-9, 381 (1979).

[7] A. Alesina, E. Spolaore, and R. Wacziarg, in Handbook of Economic Growth, edited by P. Aghion and S. N. Durlauf (North Holland, Amsterdam, 2005), Chap. 23, p. 1499.

[8] M. E. J. Newman, Phys. Rev. E 74, 036104 (2006).

[9] U. Alon, Nat. Rev. Genet. 8, 450 (2007).

[10] E. Ravasz, A. L. Somera, D. A. Mongru, Z. N. Oltvai, and A. L. Barabási, Science 297, 1551 (2002).

[11] A. Clauset, C. Moore, and M.E. J. Newman, Nature (London) 453, 98 (2008).

[12] B.S. Everitt, S. Landau, and M. Leese, Cluster Analysis (Oxford Univ. Press, New York, 2001).

[13] J. Anderson, Measurement of Protection (2009), Prepared for the Palgrave Handbook of International Trade.

[14] R. Kaesler and J. Cairns, American Midland Naturalist 88, 56 (1972).

[15] T. Friedman, The World is Flat: A Brief History of the Twenty-First Century (Farra, Straus and Giroux, New York, 2005).

[16] R. J. R. Elliott and K. Ikemoto, Asian Economic Journal 18, 1 (2004)

[17] V. Carvalho, Economics Working Papers 1206, Universitat Pompeu Fabra (2007).

[18] G. Koop, M. Pesaran, and S. Potter, J. Econometrics 74, 119 (1996).

[19] T. Abeysinghe and K. Forbes, Rev. Intl. Economics 13, 356 (2005).

[20] The Economist, "Trade, exchange rates, budget balances, and interest rates" (May 2nd, 2009), p. 98.

[21] OECD Stat. Extracts (http://stats.oecd.org/). 